\title{
Labor-Input Requirements for Experimental Production of Summer Peppers Under Drip Irrigation $^{1,2}$
}

\author{
Megh R. Goyal ${ }^{3}$
}

ABSTRACT

The total man-hours per ha requirements were $2,868.2$ and $3,029.0$, respectively in plastic mulched and non-mulched plots for summer peppers under drip irrigation. Harvesting and post harvest operations consumed $51.7 \%$ of total labor input. Plastic mulch management needed $15.3 \% \mathrm{com}-$ pared to $6.7 \%$ for installation and dismantling of the drip irrigation system. Weeding operation required 6.6 and $27.1 \%$, respectively of the labor in mulched and non-mulched plots.

\section{INTRODUCTION}

In cultivation of any crop, expenditure is incurred on various farming operations. No specific information is available to correlate the cost incurred on various operations with increase in crop production. Theoretically, the source of energy is of no consequences to farm operations so long as the job is performed satisfactorily at the proper time. ${ }^{4}$ Unaided, man can plow, sow, irrigate, harvest, and handle a crop. Practically speaking, however, the availability of non-human energy is of immense benefit in carrying out farm operations; it is cheaper and more efficient than man labor. In Puerto Rico the production of vegetable crop involves a technology which makes use of man and mechanical power as complementary inputs for most farm operations.

The crop type and associated acreage are the primary determinants of energy needs. Timeliness and intensity of operation are the essential ingredients for a successful experiment. Shortages of energy at any time will cause tardy or inadequate performance of activities ${ }^{5}$. There are two cropping seasons in Puerto Rico-summer (April to September) and

${ }^{1}$ Manuscript submitted to Editorial Board August 28, 1981.

${ }^{2}$ This study was conducted under H-326 (S-143), Southern Regional Research Project"Trickle Irrigation in Humid Regions." The author is thankful to Messers Aníbal Torres, Héctor Medrano, and William F. Allison for their constructive criticisms and suggestions. Necessary scientific help by Mr. Luis E. Rivera is duly acknowledged.

${ }^{3}$ Assistant Agricultural Engineer, Agricultural Experiment Station, Mayagüez Campus, University of Puerto Rico, Río Piedras, P. R.

${ }^{4}$ Goyal, M. R., D. M. Byg, and K. Singh, 1979. An appropriate technology for cotton production in India. Agricultural Mechanization in Asia, Japan, 10 (2): 73-78.

${ }^{5}$ Trickle Irrigation in Humid Regions-Puerto Rico. Annual Report No. 2/Puerto Rico/ Hatch 326 (S-143)/1980-81. Agricultural Experiment Station, University of Puerto Rico, Mayaguez Campus, College of Agricultural Sciences, Rio Piedras, P. R. October 1, 1980 to September 30, 1981. 
winter (October to March). The energy needs are maximum for harvesting periods of vegetable production. ${ }^{5}$

This study estimated daily, weekly, and operational labor-input requirements for experimental production of peppers under drip irrigation during summer of 1981 at Fortuna Substation, Juana Díaz.

\section{MATERIALS AND METHODS}

The field experiment ${ }^{5}$ on effects of plastic mulch and varying water application rates on pepper yield was used to estimate the operational, weekly, and daily labor requirements for experimental production of pepper (variety Cubanelle). The whole field was considered as a single unit for the purpose of this study.

Beds were shaped with a bed shaper for $1.8 \mathrm{~m}$ spacing. The 0.0015 gauge silver-coated black plastic mulch was manually placed over the desired beds. A 5-cm diameter galvanized pipe was used to punch the holes in the plastic at the desired positions. Plot size was $10.8 \times 162 \mathrm{~m}$ (90 beds, each $10.8 \mathrm{~m}$ long). Pepper plants were transplanted March 25, 1981, on both sides of a dual chamber drip line in a zigzag pattern at a distance of $15 \mathrm{~cm}$ from the drip line. The plant spacing was $30 \mathrm{~cm}$ down the row $(37,037$ plants per ha). The daily labor requirement to complete each operation was recorded. The experiment was terminated July 20, 1981.

\section{RESULTS AND DISCUSSIONS}

The data collected was analyzed (tables 1,2, and 3). Table 1 presents daily labor requirements for experimental production of peppers. A tractor was used for land preparation and spray applications on days 30, 77, 79, and 100. All other operations were completed manually. Total labor requirements during the crop season were 2,868.2 and 3,029 manhours per hectare in plastic-mulched and non-mulched plots, respectively. Tensiometer reading operation consumed 22.5 man-hours per ha. Removal and disposal of plastic mulch consumed 232.6 man-hours per ha. Labor-input requirements were highest on the 112th day.

Table 2 reveals man-hours per ha requirements for each successive week. It also indicates percentage distribution of labor input for each week. Highest percentage of labor input was 21.8 and 17.7 percent on eleventh julian week in non-mulched plots and on sixteenth julian week in mulched plots, respectively. Drip irrigation installation consumed 2.0 percent of total labor input. The layout of plastic mulch used 7.2 percent of labor input.

The man-hours per hectare requirements for each operation are indicated in table 3. It also indicates percentage distribution of labor input for each operation and frequency of operation. This table reveals that 
TABLE 1.-Daily labor requirements for experimental production of peppers (var. Cubanelle) under drip irrigation. Date of transplanting: March 25, 1981. Date of last harvest: July 15, $81^{1}$

\begin{tabular}{|c|c|c|c|}
\hline \multirow{2}{*}{$\begin{array}{l}\text { Julian } \\
\text { day }\end{array}$} & \multirow{2}{*}{ Operation } & \multicolumn{2}{|c|}{ Labor input, man-hr/ha } \\
\hline & & Plastic & No plastic \\
\hline 一 & Disc harrowing (Ford 5000) & 6.5 & 6.5 \\
\hline - & Bed shaping (Ford 5000); Bed apart $=1.8 \mathrm{~m}$ & 2.8 & 2.8 \\
\hline - & Layout main, submains, manifold and mark the field & 5.0 & 5.0 \\
\hline- & Layout drip line & 16.2 & 16.2 \\
\hline- & Connect hook up tube to drip lines and submains & 9.3 & 9.3 \\
\hline - & Straighten drip lines and put soil to anchor drip lines & 10.8 & 10.8 \\
\hline- & Flush submains, drip lines & 4.0 & 4.0 \\
\hline - & $\begin{array}{l}\text { Close ends of drip lines ( } 180 \text { connections) and sub- } \\
\text { mains }\end{array}$ & 12.1 & 12.1 \\
\hline - & Put markers to mark each line & 1.0 & 1.0 \\
\hline - & Layout plastic mulch & 155.1 & - \\
\hline - & Punch holes in plastic mulch & 52.6 & - \\
\hline- & Transport seedlings from Cabo Rojo & 4.0 & 4.0 \\
\hline 00 & Transplant pepper seddlings & 166.1 & 166.1 \\
\hline 01 & Soil treatment (back sprayer) & 27.6 & 27.6 \\
\hline 02 & Tensiometer installation & 3.0 & 3.0 \\
\hline 05 & Fertilization & 29.4 & 18.8 \\
\hline 05 & Retransplant dead spots & 57.7 & 45.4 \\
\hline 07 & Tensiometer station identification & 1.5 & 1.5 \\
\hline 14 & Weeding, hand hoe & - & 105.2 \\
\hline 26 & Weeding, hand hoe & 77.5 & - \\
\hline 27 & Spraying, back sprayer & 22.5 & 22.5 \\
\hline 30 & Spraying, Ford 3000 & 5.4 & 5.4 \\
\hline 35 & Weeding, hand hoe & - & 304.6 \\
\hline 41 & Fertilization, 10:10:8 & 29.4 & 18.8 \\
\hline 42 & Spraying, back sprayer & 27.6 & 27.6 \\
\hline 47 & Spraying, back sprayer & 22.5 & 22.5 \\
\hline 50 & Weeding, hand hoe & 44.3 & 155.1 \\
\hline 54 & Spraying, back sprayer & 27.6 & 27.6 \\
\hline 58 & Harvesting & 290.3 & 290.3 \\
\hline 58 & Grading, handling and weighing & 92.2 & 92.2 \\
\hline 62 & Spraying, back sprayer & 27.6 & 27.6 \\
\hline 76 & Weeding, hand hoe & 66.5 & 254.8 \\
\hline 77 & Harvesting & 253.5 & 253.5 \\
\hline 77 & Grading, handling, weighing & 138.2 & 138.2 \\
\hline 77 & Spraying, Ford 3000 & 11.1 & 11.1 \\
\hline 79 & Spraying, Ford 3000 & 8.3 & 8.3 \\
\hline 85 & Spraying, back sprayer & 22.5 & 22.5 \\
\hline 86 & Checking drip lines & - & 13.8 \\
\hline 92 & Harvesting & 149.8 & 149.8 \\
\hline 92 & Grading, handling, weighing & 55.3 & 55.3 \\
\hline 93 & Fertilization, 10:10:8 & 29.4 & 18.8 \\
\hline 100 & Spraying, Ford 3000 & 8.3 & 8.3 \\
\hline 112 & Harvesting & 354.8 & 354.8 \\
\hline 112 & Grading, handling, weighing & 149.8 & 149.8 \\
\hline
\end{tabular}


TABLE 1.-(cont.)

\begin{tabular}{|c|c|c|c|}
\hline \multirow{2}{*}{$\begin{array}{l}\text { Julian } \\
\text { day }\end{array}$} & \multirow{2}{*}{ Operation } & \multicolumn{2}{|c|}{ Labor input, man-hr/ha } \\
\hline & & Plastic & No plastic \\
\hline \multirow[t]{4}{*}{117} & Dismantling the irrigation system & & \\
\hline & $\begin{array}{l}\text {-Collect drip lines, submains, main, manifold, } \\
\text { hookup tube }\end{array}$ & 130.0 & 130.0 \\
\hline & -Remove and clean moisture cells & 4.0 & 4.0 \\
\hline & -Remove and dispose plastic & 232.6 & - \\
\hline \multirow[t]{2}{*}{ - } & Reading; repair and servicing moisture sensors & 22.5 & 22.5 \\
\hline & TOTAL & 2868.2 & 3029.0 \\
\hline
\end{tabular}

${ }^{1}$ Bed spacing was $1.8 \mathrm{~m}$. Pepper seedlings were transplanted on both sides of a dual chamber drip line and were spaced $30 \mathrm{~cm}$ down the row $(37,037$ plants per ha).

TABLE 2.-Weekly labor requirements for experimental production of peppers (var. Cubanelle) under drip irrigation. Date of transplanting: March 25, 81. Date of last harvest: July 15, 81

\begin{tabular}{|c|c|c|c|c|c|}
\hline \multirow{3}{*}{$\begin{array}{l}\text { Week } \\
\text { No. }\end{array}$} & \multirow[b]{3}{*}{ Operation } & \multicolumn{4}{|c|}{ Labor requirements } \\
\hline & & \multicolumn{2}{|c|}{ Plastic } & \multicolumn{2}{|c|}{ No plastic } \\
\hline & & $\begin{array}{l}\text { Man-hr } \\
\text { per ha }\end{array}$ & $\begin{array}{l}\text { Percent } \\
\text { of total }\end{array}$ & $\begin{array}{l}\text { Man-hr } \\
\text { per ha }\end{array}$ & $\begin{array}{l}\text { Percent } \\
\text { of total }\end{array}$ \\
\hline- & Land preparation & 9.3 & 0.3 & 9.3 & 0.3 \\
\hline- & Installation drip irrigation system & 58.4 & 2.0 & 58.4 & 1.9 \\
\hline- & Layout plastic mulch & 207.7 & 7.2 & - & - \\
\hline- & Transportation of seedlings & 4.0 & 0.1 & 4.0 & 0.1 \\
\hline 01 & $\begin{array}{l}\text { Transplanting, fertilization, soil } \\
\text { treatment, tensiometer installa- } \\
\text { tion }\end{array}$ & 285.3 & 9.9 & 262.4 & 8.7 \\
\hline 02 & Weeding & - & - & 105.2 & 3.5 \\
\hline 04 & Weeding, spraying & 100.0 & 3.5 & 22.5 & 0.7 \\
\hline 05 & Spraying, weeding & 5.4 & 0.2 & 310.0 & 10.2 \\
\hline 06 & Spraying, fertilization & 57.0 & 2.0 & 46.4 & 1.5 \\
\hline 07 & Spraying & 22.5 & 0.8 & 22.5 & 0.7 \\
\hline 08 & Weeding, spraying & 71.9 & 2.5 & 182.7 & 6.0 \\
\hline 09 & Harvesting, spraying & 410.1 & 14.3 & 410.1 & 13.5 \\
\hline 11 & Weeding, harvesting, spraying & 469.3 & 16.5 & 657.6 & 21.8 \\
\hline 12 & Spraying & 8.3 & 0.3 & 8.3 & 0.3 \\
\hline 13 & Spraying, drip line checking & 22.5 & 0.8 & 36.3 & 1.2 \\
\hline 14 & Harvesting, fertilization & 234.5 & 8.2 & 223.9 & 7.4 \\
\hline 15 & Spraying & 8.3 & 0.3 & 8.3 & 0.3 \\
\hline 16 & Harvesting & 504.6 & 17.7 & 504.6 & 16.7 \\
\hline 17 & $\begin{array}{l}\text { Dismantling irrigation system, re- } \\
\text { moval of plastic }\end{array}$ & 389.1 & 13.4 & 156.5 & 5.2 \\
\hline & TOTAL & 2868.2 & 100.0 & 3029.0 & 100.0 \\
\hline
\end{tabular}


harvesting, grading, product handling, and weighing operations consumed 51.7 percent of total labor input and 1483.9 man-hours were required to complete these operations. The weeding operation consumed 27.1 and 6.6 percent of total labor input in non-mulched and mulched plots respectively. Which is equivalent of 819.7 and 188.3 man-hours per ha in nonmulched and mulched plots respectively. Installation and dismantling of drip irrigation system required 58.4 and 134.0 man-hours per ha respec-

TABLE 3.-Operational labor requirements for experimental production of peppers (variety Cubanelle) under drip irrigation. Date of transplanting: March 25, 1981. Date of last harvest: July 15, 81

\begin{tabular}{|c|c|c|c|c|c|}
\hline \multirow[b]{3}{*}{ Operation } & \multirow[b]{3}{*}{ Frequency } & \multicolumn{4}{|c|}{ Labor requirements } \\
\hline & & \multicolumn{2}{|c|}{ Plastic } & \multicolumn{2}{|c|}{ No plastic } \\
\hline & & $\begin{array}{l}\text { Man-hr } \\
\text { per ha }\end{array}$ & $\begin{array}{l}\text { Percent } \\
\text { of total }\end{array}$ & $\begin{array}{l}\text { Man-hr } \\
\text { per ha }\end{array}$ & $\begin{array}{l}\text { Percent } \\
\text { of total }\end{array}$ \\
\hline Land preparation & 1 & 9.3 & 0.3 & 9.3 & 0.3 \\
\hline Installation of drip irrigation system & 1 & 58.4 & 2.0 & 58.4 & 1.9 \\
\hline \multicolumn{6}{|l|}{ Layout of plastic mulch and punching } \\
\hline of holes & 1 & 207.7 & 7.2 & - & - \\
\hline Transplanting & 1 & 223.8 & 7.8 & 211.5 & 7.0 \\
\hline Soil treatment at planting & 1 & 27.6 & 1.0 & 27.6 & 0.9 \\
\hline \multicolumn{6}{|l|}{ Tension measurement-installation, } \\
\hline operation, reading, service, etc. & - & 27.0 & 1.0 & 27.0 & 0.9 \\
\hline Fertilization & 3 & 88.2 & 3.1 & 56.4 & 1.9 \\
\hline Spraying & 10 & 183.4 & 6.4 & 183.4 & 6.1 \\
\hline \multicolumn{6}{|l|}{ Weeding } \\
\hline -Mulched plots & 3 & 188.3 & 6.6 & - & - \\
\hline -Non mulched plots & 4 & - & - & 819.7 & 27.1 \\
\hline Harvesting & 4 & 1048.4 & 36.5 & 1048.4 & 34.5 \\
\hline Grading, product handling, weighing & 4 & 435.5 & 15.2 & 435.5 & 14.4 \\
\hline Dismantling of drip irrigation system & 1 & 134.0 & 4.7 & 134.0 & 4.4 \\
\hline Removal and disposal of plastic & 1 & 232.6 & 8.1 & - & - \\
\hline \multicolumn{6}{|l|}{ Miscellaneous } \\
\hline -Transport seedlings & 1 & 4.0 & 0.1 & 4.0 & 0.1 \\
\hline -Checking drip lines & 1 & - & - & 13.8 & 0.5 \\
\hline TOTAL & & 2868.2 & 100.0 & 3029.0 & 100.0 \\
\hline
\end{tabular}

tively. Plastic mulch layout and disposal operations used 207.7 and 232.6 man-hours per ha respectively. Transplanting consumed 223.8 and 211.5 man-hours per ha in mulched and non-mulched plots. Man-hours per ha requirements for the spraying operations were 183.4 .

\section{RESUMEN}

El requerimiento total de hombres-hora por hectárea fue 2,868.2 y 3,029 .0 en los predios con cubierta y sin cubierta plástica, respectiva- 
mente, para pimientos regados por goteo durante el verano. La labor antes y después de la cosecha requirió el 51.7 por ciento de la labor total empleada. La labor requerida por la cubierta plástica fue 15.3 por ciento en contraste con 6.7 por ciento para la instalación y desmantelamiento del sistema de riego por goteo. Los desyerbos requirieron 6.6 y 27.1 por ciento de la labor total empleada en los predios con cubierta y sin cubierta plástica, respectivamente. 